\title{
Percepciones sobre la Policía en un grupo de países iberoamericanos: relaciones directas y mediadoras con el miedo al crimen, la victimización y la eficacia colectiva
}

\author{
Perceptions of the Police in a group of Latin American countries: direct and \\ mediating relations with the fear of crime, victimization and collective efficacy
}

Percepções sobre a Polícia em um grupo de países ibero-americanos: relações diretas e mediadoras com o medo do crime, a vitimização e a eficácia coletivaa

José Ignacio Ruiz-Pérez

http://orcid.org/0000-0002-8996-9736

Universidad Nacional de Colombia, Bogotá, Colombia

- Fecha de recepción: 2019-07-08

- Fecha concepto de evaluación: 2019-09-20

- Fecha de aprobación: 2019-1I-04 http://dx.doi.org/ | 0.22335/rlct.v I Ii3.968
Para citar este artículo / To reference this article / Para citar este artigo: Ruiz-Pérez, J. I. (2019). Percepciones sobre la Policía en un grupo de países iberoamericanos: relaciones directas y mediadoras con el miedo al crimen, la victimización y la eficacia colectiva. Revista Logos Ciencia \& Tecnología, I I (3): 195-205. http://dx.doi.org/ I0.22335/rlct.v I Ii3.968

\section{RESUMEN}

El objetivo del presente trabajo fue conocer las relaciones entre las percepciones sobre la Policía y el miedo al crimen, incluso el papel mediador de estas en las relaciones entre la victimización criminal y la eficacia colectiva sobre el miedo al crimen. Participaron muestras no aleatorias de estudiantes universitarios y profesionales de doce países latinoamericanos. Los resultados mostraron alta fiabilidad de las medidas de percepciones sobre la Policía ( $\alpha$ de Cronbach entre 0,84 y 0,91), del miedo al crimen $(0,7|-0,9|)$ y de la eficacia colectiva $(0,64-0,9 \mid)$. Como resultados se encontró a) correlaciones directas y significativas entre las percepciones sobre la Policía y el miedo al crimen en seis muestras nacionales, b) correlaciones directas entre victimización y miedo en cuatro países, c) dos correlaciones directas y dos inversas entre percepciones sobre la Policía y victimización, e) un efecto mediador significativo de la victimización entre el miedo al crimen y la satisfacción con la Policía, f) la eficacia colectiva mostró correlaciones directas con las percepciones sobre la Policía en tres muestras de cinco y g) por países, las muestras de Guatemala, Bolivia y Venezuela mostraron los niveles más altos de miedo al crimen y una peor percepción sobre la Policía

Palabras clave: Policía, crimen, seguridad, percepción, criminalidad 


\begin{abstract}
SUMMARY
The objective of this paper is to understand the relationship between perceptions of the Police and the fear of crime, including their mediating role in the relationship between criminal victimization and collective efficacy related to the fear of crime. Non-random samples of university students and professionals from twelve Latin American countries participated.The results showed high reliability of the measures of perceptions regarding the Police ( $\alpha$ of Cronbach between 0.84 and 0.91 ), fear of crime $(0.7 \mathrm{I}-0.9 \mathrm{I})$ and collective efficacy $(0.64-0.9 \mathrm{I})$. The results were: a) direct and significant correlations between the perceptions of the Police and the fear of crime in six national samples, b) direct correlations between victimization and fear in four countries, c) two direct and two inverse correlations between perceptions of the Police and victimization, e) a significant mediating effect of victimization between fear of crime and satisfaction with the Police, ff collective efficacy showed direct correlations with perceptions of the Police in three out of five samples, and g) by countries, samples from Guatemala, Bolivia andVenezuela showed the highest levels of fear of crime and a worse perception of the Police
\end{abstract}

Keywords: police, crime, security, perception, criminality

\title{
SUMÁRIO
}

O objetivo deste trabalho foi conhecer as relações entre as percepções sobre a polícia e o medo do crime, inclusive o papel mediador destas nas relações entre a vitimização criminal e a eficácia coletiva sobre o medo do crime. Participaram amostras não aleatórias de estudantes universitários e profissionais de doze países latino-americanos. Os resultados mostraram uma alta confiabilidade das medidas de percepções sobre a Polícia ( $\alpha$ de Cronbach entre 0,84 e 0,9I), do medo do crime $(0,7 \mid-0,9 I)$, e da eficácia coletiva $(0,64-0,91)$. Como resultados, obteve-se a) correlações diretas e significativas entre as percepções sobre a polícia e o medo do crime em seis amostras nacionais, b) correlações diretas entre vitimização e medo em quatro países, c) duas correlações diretas e duas inversas entre percepções sobre a Polícia e vitimização, e) um efeito mediador significativo da vitimização entre o medo do crime e a satisfação com a Polícia,f) a eficácia coletiva mostrou correlações diretas com as percepções sobre a polícia em três amostras de cinco e g) por países, as amostras da Guatemala, Bolívia e Venezuela revelaram os níveis mais altos de medo do crime e uma pior percepção sobre a polícia.

Palavras-chave: Polícia, crime, segurança, percepção, criminalidade

De acuerdo con Cruz (2010), las prácticas de la policía constituyen la primera percepción para la mayoría de los ciudadanos acerca de cómo el Estado responde a los problemas cotidianos, ya que es la primera autoridad a la que se suele recurrir ante problemas sociales, incluyendo emergencias. En ese marco, las percepciones y emociones sobre la Policía hacen parte de lo que se ha denominado la dimensión subjetiva de la seguridad ciudadana (Páez, Peón \& Ramírez, 2018). Esta incluye el miedo al crimen y debe tener una consideración y un abordaje específico, diferente del combate de la criminalidad objetiva (Ruiz, 2014), ya que los niveles de inseguridad no guardarían relación directa necesariamente con las tasas reales de victimización (Meško, Fallshore, Rep \& Huisman, 2007).
De esta manera, es preciso conocer qué variables inciden en el temor al crimen y contribuyen a su aumento $o$ a su reducción, puesto que una alta percepción de inseguridad se relaciona con consecuencias en la salud psicológica (Alfaro, Puente, Da Costa, Ruvalcaba \& Páez, 20I8), en la manera en que las personas tratan de evitar ser víctimas del crimen (Martínez-Zelaya, Muratori \& Ruiz, 2014) y en un posible deterioro del apoyo a la democracia, debido a que la ciudadanía puede optar por alternativas políticas que prometen seguridad a costa de reducir libertades (Páez et al., 2018) cuando se percibe alta inseguridad y niveles elevados de crimen. Así, el contexto actual de América Latina se caracterizó en 2018 por niveles de criminalidad muy elevados, con un 8 \% de la población mun- 
dial y un $33 \%$ de homicidios, y con niveles de inseguridad altísimos, según la experta Lucía Dammert, entrevistada por Focás (2018), aunque con diferencias entre países, asociadas en parte al tipo de estructura policial (unitariacentralizada vs. descentralizada) de cada sociedad (Aparicio, 2017).

En este marco, se ha estudiado los componentes que constituyen las actitudes, emociones y percepciones sociales sobre la institución policial y sus miembros. En relación con ello, se han considerado aspectos como los recursos o equipamiento de la Policía, la eficacia en su tarea, la rapidez de reacción, el grado de amabilidad y equidad en el trato a los ciudadanos o la honestidad. Por ejemplo, se ha encontrado en Colombia una percepción de mal desempeño debido a la falta de recursos económicos (Páez et al., 2018), aunque en trabajos anteriores se halló que el nivel de equipamiento era uno de los aspectos mejor valorados por una muestra de sujetos colombianos (Ruiz, 2007). En cuanto a la eficacia, es una de las dimensiones del actuar policial más abordados (Bourne, 2016; Domanick, 2016; Focás, 2018), en el sentido de que la ciudadanía busca ser protegida de la delincuencia, a la par que desea una policía que actúe rápido (Moore \& Trojanowicz, 1988; Meško et al., 2007) y que otorgue un trato amable y equitativo (Bourne, 2016; Domanick, 2016). En este sentido, se ha encontrado que tener experiencias positivas de contacto directo con la Policía se asocia con una valoración más positiva de esta institución frente a no tener experiencias directas o que estas sean negativas (Ruiz, 2007).

Por otro lado, diferentes fuentes muestran una percepción de baja honestidad policial en varios países de América Latina. Por ejemplo, en 2006 , se encontró que la honestidad era el segundo aspecto de la Policía evaluado más negativamente, después de la velocidad de reacción, por parte de sujetos colombianos (Ruiz, 2007), y en 2008, en nueve países cubiertos por el Latinobarómetro (Cruz, 2010), más del $50 \%$ de las personas encuestadas consideraba que la Policía de su país estaba involucrada en el crimen, sobre todo en Guatemala, Bolivia y Venezuela, con más del $60 \%$ de los encuestados. Para el Latinobarómetro de 2018 , el porcentaje promedio de confianza en la Policía fue del $35 \%$, se mantuvo en el mismo nivel de 2017, y con menor confianza entre los ciudadanos de México, Nicaragua y El Salvador; en la misma línea, el $45 \%$ creían que todos o casi todos los policías estaban involucrados en el crimen, frente al $46 \%$ que consideraban que ninguno o algunos (Latinobarómetro, 2018). Para el periodo de 1995 a 2016, el promedio de confianza en la Policía ha sido en las sociedades latinoamericanas del $36 \%$ de los encuestados, por encima del Gobierno, el Congreso, los partidos políticos o el poder judicial, y por debajo de la Iglesia católica o de las Fuerzas Armadas. Este promedio es inferior al encontrado en la Unión Europea (UE), con el $75 \%$ de favorabilidad y en África, con el $53 \%$ (Casas, González \& Mesías, 20I8). Estos últimos autores indican que la percepción de corrupción en la Policía fue más alta en Bolivia, Guatemala, México, Perú, República Dominicana y Venezuela, y más baja en Chile, Ecuador, Nicaragua y Uruguay.

Las relaciones entre la percepción sobre la Policía y la percepción de inseguridad han sido investigadas de diversas maneras. Un abordaje frecuente es considerar que una percepción de baja eficacia de la Policía se asocia con un mayor temor al delito (Alda, Bennett \& Morabito, 2017; Bourne, 2016; Meško et al., 2007; Ruiz, 20I4; Weisburd \& Eck, 2004). Ahora, también se ha considerado el nivel de miedo al delito como un factor predictor de la satisfacción con la Policía (Johnson, 2016), de modo que el temor al crimen estaría determinado por diferentes variables, de nivel individual como la victimización (Alda et al., 2017), el género o la edad (Bourne, 2016; Meško et al., 2007), o por variables de nivel comunitario como la pobreza, la tasa de desempleo, la cohesión social, la tasa de criminalidad en el sector, entre otras (Alda et al., 2017). En cuanto a la cohesión social, se ha propuesto el constructo de eficacia colectiva como un tipo de relaciones comunitarias en las que la gente se involucra activamente en resolver problemas de la comunidad y en cuidar preferencialmente de los grupos sociales más vulnerables, encontrándose que los entornos con mayor eficacia colectiva presentan menos tasas de criminalidad y menos percepción de inseguridad (Sampson, 2003, citado por Espinosa-Poveda \& Ruiz-Pérez, 20I2).Alda et al. (2017) encuentran, además, que la percepción o satisfacción con la Policía tiene un efecto mediador sobre el miedo al crimen en relación con estas variables de nivel comunitario.

Por otro lado, Bourne (2016) indica que unas u otras relaciones entre percepción de la Policía y el miedo al crimen arrojan resultados contradictorios $o$ incluso nulos en la literatura, por lo que los objetivos de este trabajo son a) conocer las relaciones entre la percepción sobre la Policía y el miedo al crimen en muestras de doce países iberoamericanos, b) conocer las relaciones entre victimización criminal y percepción sobre la Policía y c) 
conocerlas relaciones entrelas variablesanteriores-miedo, victimización y percepción sobre la Policía- con una variable de índole comunitaria: la eficacia colectiva.

\section{Metodología}

\section{Participantes}

Los participantes son 2656, procedentes de muestras no aleatorias de doce países iberoamericanos: Colombia $(n=1320$, entre 2006 y 20|4), El Salvador $(n=107$, entre 2007 y 2008), España $(n=739$, entre 2007, 2009 y 20I2), Perú ( $n=103$, en 2008), Argentina $(n=91$, en 2007), México ( $n=486$, entre 2008 y 20I4), Costa Rica $(n=77$, en 2009), Guatemala ( $n=94$, en 2009), Bolivia $(n=66$, en 2009), Paraguay ( $n=66$, en 2008), Venezuela $(n=5 \mathrm{I}$, en 20I3) y Brasil ( $n=74$, en 20I4). En general, las muestras están conformadas por estudiantes universitarios que respondieron el instrumento en forma de aplicación colectiva en salones de clase, excepto para el caso de las muestras de Venezuela y Brasil, conformadas por asistentes a respectivos congresos de psicología.

En la tabla I, se presentan algunos estadísticos descriptivos de la muestras en aspectos sociodemográficos básicos. Dado que la mayoría de los participantes son estudiantes y jóvenes, es lógico que gran parte son solteros. Las medias de edad oscilan entre los 20 años para los participantes de Cuzco, y casi los 29, para los de Colombia. Por otro lado, la mayoría son mujeres en todas las muestras, excepto en el caso de la muestra de Guatemala y de Bolivia, con mayoría de sujetos varones. En cuanto al nivel socioeconómico, la clase social más frecuente es la media, seguida de la clase baja y la clase alta en proporciones semejantes para la mayoría de los países, excepto para los participantes de Colombia, El Salvador y España, con mayor proporción de sujetos de clase baja que de clase alta.

\section{Materiales e instrumentos}

Los participantes respondieron un cuestionario estructurado en el formato de batería conformada por las siguientes escalas:

- Escala de percepción de la Policía (Ruiz, 2007). Esta escala consta de ocho ítems con cuatro opciones de respuesta desde I (nada) a 4 (siempre) sobre otros tantos aspectos percibidos en la actuación policial, como la eficacia, la presencia en el sector, la velocidad de reacción, la honestidad, el cumplimiento de sus deberes, el equipamiento, la preocupación de la Policía por el bien común y el nivel de formación. De la escala se extrae un puntaje total, que oscila entre I y 4 , al sumar la respuesta a cada ítem y dividir el resultado entre el número de ítems. La fiabilidad interna de la escala ha mostrado ser adecuada, entre 0,88 (Ruiz, 2007) y 0,86 (Ruiz \& Turcios, 2009). Las respuestas a dicha escala pueden ser analizadas ítem a ítem, y también en la sumatoria de los ocho ítems, que da lugar a un puntaje en satisfacción con la Policía, que oscila entre 8 y 32, es decir, de menor a mayor satisfacción con la Policía.

- Escala de miedo al crimen (Páez \& Ruiz, 2012). Esta escala consta de seis ítems sobre el temor que da pasear en la

Tabla I. Características sociodemográficas de la muestra por cada país

\begin{tabular}{|lcccc|}
\hline \multicolumn{1}{c}{ País } & \% mujeres & Edad (IC 95 \%) & \% Solteros/casados o unión libre & Clase social: Baja Media Alta \\
\hline Colombia & 54,5 & $28,6(27,9-29,3)$ & $66,0-29,5$ & $25,6-71,4-3,0$ \\
\hline El Salvador & 56,1 & $27,3(26,0-28,6)$ & $74,6-23,7$ & $24,0-68,3-7,7$ \\
\hline España & 59,7 & $21,9(21,5-22,2)$ & $81,7-16,7$ & $18,0-74,3-7,7$ \\
\hline Perú & 56,3 & $20,2(19,6-20,7)$ & $92,2-6,8$ & $10,7-77,7-11,7$ \\
\hline Argentina & 75,8 & $21,6(20,9-22,3)$ & $9,3-87,4-6,3$ \\
\hline México & 56,8 & $21,2(20,9-21,5)$ & $93,6-6,4$ & $9,3-84,4-6,3$ \\
\hline Costa Rica & 66,2 & $20,6(19,7-20,6)$ & $94,6-4,4$ & $9,3-81,3-9,3$ \\
\hline Guatemala & 18,1 & $20,9(20,5-21,3)$ & $100-0$ & $15,1-73,1-11,8$ \\
\hline Bolivia & 24,5 & $22,0(20,7-23,4)$ & $97,9-1,1$ & $6,0-88,0-6,0$ \\
\hline Paraguay & 68,2 & $27,2(25,2-29,2)$ & $90,7-5,6$ & $9,6-80,8-9,6$ \\
\hline Venezuela & 66,7 & $26,2(23,5-28,9)$ & $75,3-22,1$ & $64,7-13,7$ \\
\hline Brasil & 49,3 & $23,6(22,4-24,9)$ & $82,9-17,2$ & \\
\hline
\end{tabular}


zona de residencia de noche, ser víctima de un delito, ser víctima de un delito dentro del hogar y el temor al barrio, la localidad o distrito, y la ciudad. Cada ítem presenta cuatro alternativas de respuesta, sobre cuánto temor genera la situación representada en el ítem, desde I (nada) a 4 (siempre). La fiabilidad de la escala ha oscilado adecuada en diferentes estudios, desde 0,80 (Ruiz, 2007) a 0,86 (Ruiz \& Turcios, 2009). Los resultados en la escala pueden ser analizados ítem a ítem o sumando las respuestas de cada sujeto a cada ítem, $y$ así se obtiene un puntaje en miedo difuso, que oscila de 6 (menos temor percibido) a 24 (más miedo percibido).

- Escala de victimización (adaptada de Ruiz, López, Norza \& Rodríguez, 2012). Esta escala consta, a los efectos de este trabajo, de una lista de siente delitos sobre los cuales se pregunta a cada participante si le ha ocurrido o no en los últimos doce meses: robo o hurto, amenaza, extorsión, secuestro, agresión física, agresión sexual y corrupción. Esta escala se sometió a un análisis factorial para reducir los factores a un solo puntaje dimensional, en el que la media es cero, por encima de ella, y a mayor victimización el puntaje es positivo; $y$ mayor y por debajo de cero y a menor puntaje, la victimización es menor para cada sujeto.

- Escala de eficacia colectiva (adaptada de Carroll, Rosson \& Zhou, 2005, citados por Espinosa \& Ruiz, 20I2). Esta escala consta de diecisiete ítems, con cuatro opciones de respuesta, desde I (total desacuerdo) a 4 (total acuerdo), sobre aspectos percibidos en la comunidad como "Puede elaborar leyes justas, a pesar de los conflictos en el país" (ítem I),"Puede mejorar la calidad de vida de la comunidad, aun cuando los recursos sean limitados o escasos" (ítem 6) o "Puede mejorar la calidad y el acceso a los servicios para personas con discapacidad" (ítem 16). Esta escala se aplicó en la versión del cuestionario de cinco de las muestras nacionales de este estudio. La escala arroja un puntaje en eficacia colectiva al sumar las respuestas de cada sujeto a cada uno de los ítems. Este puntaje puede oscilar de 17 a 68 (de menor a mayor eficacia percibida).

- Datos sociodemográficos. Recogió la edad, el género, el estrato socioeconómico de los participantes (en tres categorías: clase baja, media y alta) y el estado civil.

\section{Procedimiento, aspectos bioéticos}

\section{y plan de análisis}

Una vez confeccionado el cuestionario, se aplicó de forma colectiva por salones de clase en las universidades participantes y en los congresos académicos (Venezuela y Brasil), no sin antes solicitar y obtener las autorizaciones correspondientes. El cuestionario incluyó un formato de consentimiento informado, y se solicitó a los participantes responder de forma voluntaria la batería, a quienes se les informó del respeto al anonimato y del objetivo exclusivamente académico de la investigación y del uso co- rrespondiente de los datos. Los datos de las muestras de Brasil, Perú, Colombia, Guatemala, Costa Rica, El Salvador y Venezuela fueron recopilados por miembros del equipo de investigación autor de este trabajo. En los demás países, los datos fueron recolectados por colaboradores locales. Para los análisis, se llevó a cabo el examen descriptivo de las escalas en el puntaje total —satisfacción con la Policía, miedo al crimen y eficacia colectiva - y de cada ítem para las dos primeras escalas. Se calculó seguidamente la fiabilidad interna de los tres instrumentos y las correlaciones bivariadas intrapaís con dichas escalas y el indicador de victimización criminal. Por último, para el conjunto de la muestra, se llevó a cabo un análisis de mediación entre el miedo al crimen como variable predictora, la percepción de la Policía como variable criterio y el factor de victimización como variable mediadora, junto con el género y el nivel socioeconómico como variables complementarias. Por último, se calculó el tamaño de efecto de la variable género.

\section{Resultados}

En primer lugar, en la tabla 2, se presentan los estadísticos descriptivos - media y desviación típica - en cada ítem de la escala de percepción de la Policía por cada uno de los países. También se presentan los mismos estadísticos para la escala de miedo difuso al crimen, por cada muestra nacional. Se puede apreciar, a la luz de estas medias, que los aspectos de eficacia y de honestidad de la Policía son los que generan mayor insatisfacción, y en relación con el miedo al delito, ser víctima de un delito y el delito en la ciudad son los aspectos que generan mayor temor.

A continuación, se procedió a explorar la fiabilidad interna de las escalas de percepción sobre la Policía, miedo al crimen y eficacia colectiva, incluidas en este estudio, y se encontraron coeficientes satisfactorios por cada país, aunque con ciertas diferencias.Así, la escala de percepción de la Policía presenta una fiabilidad interna entre 0,7 I para la muestra argentina y 0,9l para la de El Salvador, aunque coeficientes de Cronbach superiores a 0,80 en ocho de las doce muestras. Por su lado, la escala de miedo difuso al crimen presenta coeficientes de fiabilidad interna iguales o superiores a 0,84 , salvo en el caso peruano, con un alfa de Cronbach de 0,78. En cuanto a la escala de eficacia colectiva, se encuentran coeficientes satisfactorios, por encima de 0,80 en cuatro de las cinco muestras nacionales; llama la atención el bajo índice de la escala de eficacia colectiva para la muestra de España. 
Tabla 2. Medias y desviaciones típicas de los ítems de la escala de percepción de la Policía por países

\begin{tabular}{|c|c|c|c|c|c|c|c|c|}
\hline & \multicolumn{8}{|c|}{ Sobre la Policía, en qué grado está satisfecho con su... } \\
\hline & Eficacia & $\begin{array}{l}\text { Presencia } \\
\text { en sector }\end{array}$ & $\begin{array}{l}\text { Cumple } \\
\text { deberes }\end{array}$ & $\begin{array}{l}\text { Velocidad } \\
\text { de reacción }\end{array}$ & $\begin{array}{l}\text { Preocupación } \\
\text { por bien común }\end{array}$ & $\begin{array}{l}\text { Nivel de } \\
\text { formación }\end{array}$ & Equipos & Honestidad \\
\hline Colombia & $\mathrm{I}, 77(0,74)$ & $\mathrm{I}, 87(0,8 \mathrm{I})$ & $1,93(0,70)$ & $1,66(0,76)$ & $1,90(0,73)$ & $\mathrm{I}, 99(0,8 \mathrm{I})$ & $2,38(0,84)$ & I,78 $(0,75)$ \\
\hline El Salvador & $1,62(0,67)$ & $\mathrm{I}, 73(0,73)$ & $\mathrm{I}, 88(0,68)$ & $1,63(0,76)$ & $\mathrm{I}, 77(0,68)$ & $\mathrm{I}, 85(0,75)$ & $2,04(0,77)$ & $\mathrm{I}, 64(0,64)$ \\
\hline España & $2,32(0,68)$ & $2,48(0,91)$ & $2,77(0,87)$ & $2,47(0,87)$ & $2,8 \mathrm{I}(0,86)$ & $2,84(0,84)$ & $3,02(0,77)$ & $2,58(8,37)$ \\
\hline Perú & $1,93(0,63)$ & $2,19(0,79)$ & $2, I I(0,74)$ & I,98 $(0,78)$ & $2,20(0,76)$ & $2,10(0,73)$ & $2, \mathrm{II}(0,77)$ & I,87 $(0,77)$ \\
\hline Argentina & $\mathrm{I}, 57(0,54)$ & $\mathrm{I}, 88(0,78)$ & $\mathrm{I}, 86(0,53)$ & $\mathrm{I}, 72(0,62)$ & $\mathrm{I}, 80(0,5 \mathrm{I})$ & I,97 $(0,68)$ & $2,12(0,64)$ & $\mathrm{I}, 66(0,56)$ \\
\hline México & I,84 $(0,69)$ & $\mathrm{I}, 99(0,8 \mathrm{I})$ & I,97 $(0,62)$ & $\mathrm{I}, 8 \mathrm{I}(0,75)$ & I,99 $(0,67)$ & I,87 $(0,7 \mathrm{I})$ & $2,00(0,76)$ & I,77 $(0,68)$ \\
\hline Costa Rica & I,76 $(0,58)$ & $\mathrm{I}, 77(0,7 \mathrm{I})$ & I,9I $(0,60)$ & I,75 $(0,72)$ & $2,03(0,66)$ & $2,00(0,75)$ & $\mathrm{I}, 97(0,72)$ & $\mathrm{I}, 82(0,6 \mathrm{I})$ \\
\hline Guatemala & I,33 $(0,52)$ & I,7I $(0,78)$ & I,7I $(0,52)$ & $\mathrm{I}, 44(0,54)$ & $\mathrm{I}, 64(0,60)$ & I,66 $(0,66)$ & I,92 $(0,72)$ & $\mathrm{I}, 48(0,68)$ \\
\hline Bolivia & $\mathrm{I}, 47(0,64)$ & $\mathrm{I}, 49(0,7 \mathrm{I})$ & $\mathrm{I}, 74(0,56)$ & $\mathrm{I}, 53(0,7 \mathrm{I})$ & I,86 $(0,54)$ & $\mathrm{I}, 74(0,6 \mathrm{I})$ & $\mathrm{I}, 80(0,72)$ & $\mathrm{I}, 47(0,60)$ \\
\hline Paraguay & I,68 $(0,67)$ & $\mathrm{I}, 8 \mathrm{I}(0,87)$ & I,85 $(0,67)$ & I,59 $(0,8 \mathrm{I})$ & I,79 (0,7I) & I,86 $(0,78)$ & $\mathrm{I}, 67(0,75)$ & I,7I $(0,74)$ \\
\hline Venezuela & $\mathrm{I}, 48(0,6 \mathrm{I})$ & I,52 $(0,84)$ & $\mathrm{I}, 74(0,60)$ & $\mathrm{I}, 42(0,67)$ & I,65 $(0,66)$ & $\mathrm{I}, 82(0,67)$ & $\mathrm{I}, 98(0,78)$ & $\mathrm{I}, 44(0,58)$ \\
\hline Brasil & I,77 $(0,59)$ & $\mathrm{I}, 88(0,68)$ & $2, I I(0,5 I)$ & I,76 $(0,6 I)$ & I,99 $(0,63)$ & $2,09(0,72)$ & $2,08(0,72)$ & $1,92(0,66)$ \\
\hline
\end{tabular}

Tabla 3. Medias y desviaciones típicas de los ítems de la escala de miedo al crimen por países

\begin{tabular}{|c|c|c|c|c|c|c|}
\hline & \multicolumn{6}{|c|}{ ¿En qué medida siente temor a...? } \\
\hline & $\begin{array}{c}\text { Andar por sector } \\
\text { de noche }\end{array}$ & $\begin{array}{l}\text { Víctima de } \\
\text { delito }\end{array}$ & $\begin{array}{l}\text { Víctima dentro } \\
\text { de casa }\end{array}$ & Al barrio & A la localidad & A la ciudad \\
\hline Colombia & $2,39(0,89)$ & $2,64(0,86)$ & I,99 (0,91) & $2,17(0,82)$ & $2,43(0,79)$ & $2,78(0,80)$ \\
\hline El Salvador & $2,77(0,96)$ & $3,39(0,76)$ & $2,73(0,96)$ & $2,53(0,90)$ & $2,66(0,86)$ & $3,18(0,80)$ \\
\hline España & I,78 $(0,78)$ & $1,90(0,74)$ & I,67 $(0,73)$ & $1,66(0,66)$ & I,70 $(0,67)$ & $\mathrm{I}, 78(0,7 \mathrm{I})$ \\
\hline Perú & $2,20(0,80)$ & $2,64(0,86)$ & $2,38(0,95)$ & $2,17(0,70)$ & $2,37(0,73)$ & $2,50(0,80)$ \\
\hline Argentina & $2,2(0,93)$ & $2,63(0,79)$ & $2,19(0,92)$ & $2,00(0,73)$ & $2,47(0,74)$ & $2,61(0,75)$ \\
\hline México & $2,18(0,84)$ & $2,53(0,84)$ & $2,20(0,91)$ & $2,02(0,79)$ & $2,15(0,75)$ & $2,39(0,77)$ \\
\hline Costa Rica & $2,63(I, 01)$ & $2,94(0,93)$ & $2,19(1,06)$ & $2,13(0,94)$ & $2,29(0,87)$ & $2,82(0,86)$ \\
\hline Guatemala & $2,52(0,98)$ & $2,97(0,88)$ & $2,15(0,90)$ & $2,22(0,72)$ & $2,23(0,72)$ & $2,89(0,84)$ \\
\hline Bolivia & $2,71(0,92)$ & $3,03(0,75)$ & $2,65(1,04)$ & $2,46(0,76)$ & $2,77(0,74)$ & $3,05(0,83)$ \\
\hline Paraguay & $2,73(1,16)$ & $2,77(1,16)$ & $2,47(I, \mid 4)$ & $2,42(I, I I)$ & $2,47(I, I I)$ & $2,65(1,04)$ \\
\hline Venezuela & $3,06(1,04)$ & $3,16(1,03)$ & $2,83(I, 12)$ & $2,57(0,99)$ & $2,69(0,99)$ & $2,98(1,00)$ \\
\hline Brasil & $2,82(1,05)$ & $3,09(0,87)$ & $2,21(0,88)$ & $2,42(0,84)$ & $2,59(0,79)$ & $2,93(0,78)$ \\
\hline
\end{tabular}

En cuanto a la validez convergente de ambas escalas, se calculó para cada país la correlación de Spearman entre los puntajes totales en cada una de las escalas (percepción de la Policía con miedo difuso al crimen). Llama la atención que, de las doce muestras nacionales, en seis se halló una correlación significativa entre mayor satisfacción con la Policía y menor miedo al delito, y una correlación tendencial en el mismo sentido para Brasil. Sin embargo, en cinco muestras, la correlación entre ambas variables no es significativa. Esta nula correlación no parece ligada a una baja fiabilidad interna de las escalas en esas cinco muestras nacionales (tabla 4).

Para el conjunto de la muestra, la correlación entre el miedo al crimen y la percepción de la Policía es r(3157): $-0,354, p<0,001$, mientras que la correlación entre la percepción de la Policía y el indicador de victimización es $r(28 \mathrm{I}):-0, I 49, \mathrm{p}<0,00 \mathrm{I}, \mathrm{y}$ entre esta última variable $y$ el miedo al crimen es $r(2868): 0,219$ con $p<0,00 I$, con lo cual se obtiene que un mayor miedo al crimen se relaciona 
Tabla 4. Fiabilidad interna, medias y desviaciones, y correlación entre las escalas de percepción de la Policía y medo al crimen por países

\begin{tabular}{|c|c|c|c|c|c|c|}
\hline País & $\alpha$ Percepción sobre la Policía & $\mathbf{X} \mathbf{y} \sigma$ & $\begin{array}{l}\alpha \text { Miedo al } \\
\text { crimen }\end{array}$ & $\overline{\mathbf{X}} \mathbf{y} \sigma$ & $\begin{array}{l}\alpha \text { Eficacia } \\
\text { colectiva }\end{array}$ & $\mathbf{X} \mathbf{y} \sigma$ \\
\hline Colombia & 0,89 & $\mathrm{I}, 9 \mathrm{I}(0,57)$ & 0,84 & $2,40(0,63)$ & 0,97 & $2,94(0,97)$ \\
\hline El Salvador & 0,91 & $\mathrm{I}, 77(0,56)$ & 0,87 & $2,89(0,67)$ & & \\
\hline España & 0,89 & $2,68(0,62)$ & 0,86 & $\mathrm{I}, 75(0,54)$ & 0,64 & $3,43(0,21)$ \\
\hline Perú & 0,77 & $2,07(0,46)$ & 0,78 & $2,37(0,56)$ & & \\
\hline Argentina & 0,71 & $\mathrm{I}, 82(0,35)$ & 0,84 & $2,35(0,6 \mathrm{I})$ & & \\
\hline México & 0,87 & $\mathrm{I}, 9 \mathrm{I}(0,5 \mathrm{I})$ & 0,85 & $2,24(0,6 \mathrm{I})$ & 0,82 & $2,74(0,39)$ \\
\hline Costa Rica & 0,84 & $\mathrm{I}, 88(0,48)$ & 0,91 & $2,50(0,79)$ & & \\
\hline Guatemala & 0,80 & $\mathrm{I}, 60(0,40)$ & 0,84 & $2,49(0,64)$ & & \\
\hline Bolivia & 0,74 & $\mathrm{I}, 60(0,37)$ & 0,88 & $2,78(0,67)$ & & \\
\hline Paraguay & 0,90 & $2,59(0,99)$ & 0,94 & $\mathrm{I}, 74(0,58)$ & & \\
\hline Venezuela & 0,86 & $\mathrm{I}, 6 \mathrm{I}(0,46)$ & 0,94 & $2,89(0,89)$ & 0,91 & $2,74(0,62)$ \\
\hline Brasil & 0,83 & I,95 $(0,43)$ & 0,89 & $2,68(0,69)$ & 0,89 & $2,78(0,47)$ \\
\hline
\end{tabular}

$+\mathrm{p}<0,10 ; \mathrm{p}<0,05 ; * \mathrm{p}<0,01 ; * * * \mathrm{p}<0,001$

Tabla 4. Correlaciones entre satisfacción con la Policía, miedo al crimen, victimización y eficacia colectiva por países

\begin{tabular}{|c|c|c|c|c|c|c|}
\hline País & $\begin{array}{l}\text { Satisfacción } \\
\text { con miedo }\end{array}$ & $\begin{array}{l}\text { Satisfacción- } \\
\text { victimización }\end{array}$ & $\begin{array}{l}\text { Satisfacción- } \\
\text { eficacia }\end{array}$ & $\begin{array}{l}\text { Victimización- } \\
\text { miedo }\end{array}$ & $\begin{array}{l}\text { Victimización- } \\
\text { eficacia }\end{array}$ & $\begin{array}{l}\text { Miedo- } \\
\text { eficacia }\end{array}$ \\
\hline Colombia & $\begin{array}{c}-0,173 * * * \\
(\mathrm{n}: 125 \mid)\end{array}$ & $\begin{array}{c}-0,111 * * \\
(n: 892)\end{array}$ & $\begin{array}{l}0,466 * * * \\
\text { (n: } 111)\end{array}$ & $\begin{array}{l}0,178 * * * \\
\text { (n: } 933)\end{array}$ & $\begin{array}{c}-0,009 \text { n.s } \\
\text { (n: 109) }\end{array}$ & $\begin{array}{c}0,112 \text { n.s } \\
\text { (n: } 111)\end{array}$ \\
\hline El Salvador & $\begin{array}{l}-0,|3| \text { n.s } \\
\text { (n: } 108)\end{array}$ & $\begin{array}{l}0,086 \text { n.s } \\
\text { (n: } 108)\end{array}$ & & $\begin{array}{l}0,212^{*} \\
(\mathrm{n}: 110)\end{array}$ & & \\
\hline España & $\begin{array}{c}-0,311 * * * \\
(\mathrm{n}: 718)\end{array}$ & $\begin{array}{l}0,127^{* *} \\
(\mathrm{n}: 721)\end{array}$ & $\begin{array}{c}-, 0007 \text { n.s } \\
(\mathrm{n}: 306)\end{array}$ & $\begin{array}{l}0,042 \text { n.s } \\
\text { (n: } 738)\end{array}$ & $\begin{array}{c}0,067 \text { n.s } \\
\text { (n: } 242)\end{array}$ & $\begin{array}{c}0,062 \text { n.s } \\
\text { (n: 306) }\end{array}$ \\
\hline Perú & $\begin{array}{l}-0,232^{*} \\
(\mathrm{n}=102)\end{array}$ & $\begin{array}{c}-0,067 \text { n.s } \\
\text { (n: 102) }\end{array}$ & & $\begin{array}{l}0,092 \text { n.s } \\
\text { (n: } 103)\end{array}$ & & \\
\hline Argentina & $\begin{array}{l}-0,04 \mid \mathrm{n} . \mathrm{s} \\
\text { (n: 87) }\end{array}$ & $\begin{array}{l}-0,152 \text { n.s } \\
\text { (n: 89) }\end{array}$ & & $\begin{array}{l}0,045 \text { n.s } \\
\text { (n: } 92)\end{array}$ & & \\
\hline México & $\begin{array}{l}0,07 \text { n.s } \\
\text { (n: } 479)\end{array}$ & $\begin{array}{l}0,156^{* *} \\
\text { (n: 489) }\end{array}$ & $\begin{array}{l}0,216^{* *} \\
\text { (n: } 145)\end{array}$ & $\begin{array}{l}0,168 * * * \\
\text { (n: } 484)\end{array}$ & $\begin{array}{l}-0,025 \text { n.s } \\
\text { (n: I45) }\end{array}$ & $\begin{array}{l}-0,020 \text { n.s } \\
\text { (n: } 145)\end{array}$ \\
\hline Costa Rica & $\begin{array}{c}-0,254^{*} \\
(\mathrm{n}: 76)\end{array}$ & $\begin{array}{c}-0,204+ \\
\text { (n: 74) }\end{array}$ & & $\begin{array}{l}0,265^{*} \\
\text { (n: 73) }\end{array}$ & & \\
\hline Guatemala & $\begin{array}{c}-0,27 I^{* *} \\
(\mathrm{n}: 91)\end{array}$ & $\begin{array}{l}-0,021 \text { n.s } \\
\text { (n: } 91)\end{array}$ & & $\begin{array}{l}0,021 \text { n.s } \\
(\mathrm{n}: 93)\end{array}$ & & \\
\hline Bolivia & $\begin{array}{c}-0,28 I^{*} \\
(\mathrm{n}: 52)\end{array}$ & $\begin{array}{c}-0,177 \text { n.s } \\
\text { (n: 209) }\end{array}$ & & $\begin{array}{l}0,168 \text { n.s } \\
\text { (n:54) }\end{array}$ & & \\
\hline Paraguay & $\begin{array}{l}-0,056 \mathrm{n} . \mathrm{s} \\
\text { (n: 74) }\end{array}$ & $\begin{array}{l}-0,105 \text { n.s } \\
\text { (n: 78) }\end{array}$ & & $\begin{array}{l}0,186 \text { n.s } \\
(\mathrm{n}: 74)\end{array}$ & & \\
\hline Venezuela & $\begin{array}{c}-0,067 \text { n.s } \\
\text { (n: } 46)\end{array}$ & $\begin{array}{c}-0,346^{*} \\
(\mathrm{n}: 48)\end{array}$ & $\begin{array}{l}0,324+ \\
(\mathrm{n}: 36)\end{array}$ & $\begin{array}{l}0,104 \text { n.s } \\
\text { (n: } 47)\end{array}$ & $\begin{array}{l}-0,183 \text { n.s } \\
\text { (n: 36) }\end{array}$ & $\begin{array}{c}0,269 \text { n.s } \\
\text { (n:36) }\end{array}$ \\
\hline Brasil & $\begin{array}{c}-0,205+ \\
\text { (n: } 73)\end{array}$ & $\begin{array}{c}-0,233+ \\
\text { (n: } 67)\end{array}$ & $\begin{array}{c}0,183 \text { n.s } \\
(\mathrm{n}: 69)\end{array}$ & $\begin{array}{c}0,401 * * \\
(\mathrm{n}: 67)\end{array}$ & $\begin{array}{c}0,00 \text { n.s } \\
\text { (n: } 62)\end{array}$ & $\begin{array}{c}-0,043 \text { n.s } \\
\text { (n: 68) }\end{array}$ \\
\hline
\end{tabular}

$+p<0,10 ; * p<0,05 ; * *<<0,01 ; * * * p<0,001$

con haber sufrido más experiencias de victimización, último, para delimitar el papel relativo del miedo al crimen mientras que más miedo al crimen y más victimización se y de la victimización criminal sobre la satisfacción con la relacionan con una menor satisfacción con la Policía. Por Policía, se realizó un análisis de mediación, el cual tomó 
como variable predictora el miedo al crimen, como mediadora la victimización, como criterio la satisfacción con la Policía y como covariable el estrato, ya que la correlación entre la clase social y la satisfacción con la Policía fue positiva y moderada, con $r(2427): 0,070, p=0,00 \mathrm{I}$. EI modelo total arrojó un coeficiente beta para el miedo al crimen beta: $-0,186$, SE: 0,164 , con t: $-1 \mathrm{I}, 33$ y $\mathrm{P}<0,00 \mathrm{I}$, con intervalos entre $-0,218$ y $-0,154$, mientras que el efecto directo del miedo al crimen sobre la satisfacción con la Policía fue semejante al efecto total, con beta: $-0,180$, SE: 0,016 y t: $-0,10.91, p<0,00 \mathrm{I}$, con límites entre $-0,212$ y $-0,147$. El efecto indirecto del miedo al crimen, mediado con el factor de victimización criminal fue beta: $-0,006$, SE: 0,002 , y límites entre $-0,01$ I y $-0,002$. Al no pasar los intervalos por cero, se considera significativo el modelo de interacción, aunque con un valor moderado, mientras que fue más clara y significativa la relación entre una mayor victimización criminal y una peor satisfacción con la Policía, con beta: $-I, 072$, SE: 0,332 y t: $-3,23$, $P<0,0 I$, con límites entre $-I, 723$ y $-0,420$. Por su lado, hay una relación del miedo al crimen sobre el factor de victimización, con beta: 0,005 , SE: 0,00 I y t: 5,12 con $p<0,00$ I, y límites entre 0,003 y 0,008. El estrato socioeconómico tuvo una relación tendencial con la satisfacción con la Policía (t: $1,68, p<0,10$ ), para un beta: 0,022, SE: 0,012 y límites entre $-0,003$ y 0,005. En cuanto al género, no hubo diferencias en el factor de victimización, $F(I, 2822)$ : 0,40 , y sí en la satisfacción con la policía, $\mathrm{F}(\mathrm{I}, 3 \mid 00)$ : 1,74 , $P<0,05$, y sobre todo en el miedo al crimen, $F(I, 3 \mid 52)$ : 14,42 , $p<0,001$, de forma que las mujeres mostraron menor satisfacción con la Policía (Media: 2,04, DT: 0,58) y más miedo al crimen (Media: 2,30, DT: 0,68) que los hombres (para satisfacción con la Policía, Media: 2,08 y DT: 0,69; para miedo al crimen: 2,2I y DT: 0,69). De hecho, el tamaño del efecto para el género fue de $0,|3|$, el cual puede ser considerado moderado, pero al corresponder a una muestra de alrededor de 1500 sujetos por grupo ( $\mathrm{n}$ hombres: I406; n mujeres: 1747) se puede confiar en que sí existe ese efecto con $p<0,05, y$ una probabilidad del $80 \%$ de detectar ese efecto, de acuerdo con las tablas de Cohen (Ledesma, Macbeth \& De Kohan, 2008).

\section{Discusión y conclusiones}

Quizá la institución policial es el primer contacto directo que la ciudadanía tiene con el sistema judicial, no solo en relación con posibles conflictos con la ley, sino con la observación de las conductas de los policías en diferentes escenarios: regulando el tráfico, patrullando, vigilando el acceso a edificios e instalaciones públicas, etc., y también a través de los medios de comunicación, con noticias sobre capturas, operativos y posibles conductas inapropiadas. La imagen, confianza, honestidad o eficacia de la Policía parece guardar relación con el grado de apoyo de la sociedad al régimen político vigente (Páez et al., 2018), de manera que, en contextos de alta criminalidad e inseguridad percibida como la región latinoamericana, con zonas de alta concentración de violencia en la mayoría de sus ciudades (Focás, 2018), es pertinente conocer cómo puede contribuir la percepción social sobre la Policía a disminuir la criminalidad subjetiva, es decir, el miedo al crimen.

Por ello, los objetivos de este estudio se han enfocado mediante el análisis de las relaciones entre cuatro variables principalmente: el miedo al crimen, la victimización, la percepción sobre la Policía y la eficacia colectiva. Los análisis se llevaron a cabo a nivel intrapaís, debido al diferente tamaño de las muestras disponibles. De esta manera, se pudo abordar el comportamiento de las relaciones entre las variables en cada contexto y evitar el riesgo de que un análisis para el conjunto combinado de las muestras, las tendencias de las muestras más grandes, opacaran las tendencias subyacentes en las más pequeñas. En los resultados, previamente se procedió a constatar que los instrumentos empleados -escalas de miedo al crimen, satisfacción con la Policía y eficacia colectiva- presentaran unas adecuadas propiedades psicométricas, aspecto que se cumple en líneas generales, con la excepción de la escala de eficacia colectiva para la muestra de España y la escala de percepciones sobre la Policía en la muestra de Argentina.

En relación con los componentes de las percepciones sobre la Policía, en este trabajo, se encuentra un mismo patrón de resultados: en nueve países de la muestra, la eficacia de la Policía es uno de los aspectos peor valorados, junto con la velocidad de reacción, en ocho países, y la honestidad, en cinco. Las muestras de Venezuela, Bolivia y Guatemala presentan los niveles más bajos de satisfacción con la Policía;este resultado llama la atención con relación a que las muestras no son representativas de sus países, pero coincide con lo reportado en el Latinobarómetro de 2018, en el cual esos mismos países reportan los niveles de percepción de corrupción en la Policía más altos; además, en esos mismos países, la confianza en la Policía era menor en 2014 (Casas et al., 2018). Se requiere un análisis en profundidad de las razones de estos resultados en cada 
uno de los países, lo cual excede las posibilidades de este artículo, pero se pueden mencionar los cambios sociopolíticos de alta intensidad que en los últimos lustros afectan a países como Bolivia y Venezuela, la alta tasa de homicidios en países como Guatemala y Venezuela (United Nations Office on Drugs and Crime [UNODC], 2019) y la influencia del tráfico ilegal de drogas, con la presencia de estructuras de narcotráfico y corruptoras de las instituciones. Al respecto, Bolivia, Guatemala y Venezuela se incluyen en una lista del gobierno de Estados Unidos como los de mayor tránsito y producción de estupefacientes (elPeriódico, 2019).

Por otro lado, el nivel de formación, en seis países, y el equipamiento, en diez, son los aspectos mejor valorados, con las muestras de España sobre todo, pero también de Paraguay y Perú. A pesar de ello, para el conjunto de muestras abarcadas en este trabajo, los niveles de satisfacción con la Policía no son altos, pues ningún promedio por muestra nacional llega al puntaje de tres (3), cuando el máximo posible de satisfacción es 4 . Al respecto, vale indicar que tener experiencias positivas con la Policía -por ejemplo, recibir un trato respetuoso y eficaz de parte de policías-, se asocia con desarrollar una valoración positiva (Ruiz, 2007).

Por otra parte, el perfil de resultados que se obtiene acerca del miedo al crimen es el siguiente: ser víctima de delito y el ámbito de la ciudad son los que generan mayor temor en el conjunto de las muestras nacionales, mientras que el temor a ser víctima de un delito dentro del hogar o en el propio barrio son los aspectos que generan menor temor. Es decir, el miedo al crimen es menor para el ámbito mejor conocido, donde pasan más tiempo las personas, como el barrio (Meško et al., 2007; Ruiz, 20 I4). En general, las muestras españolas son las que expresan menor miedo difuso al delito, junto con la argentina (sobre miedo a pasear de noche por el lugar de residencia y temor al barrio) y la mexicana (en el temor al barrio, a la localidad y a ser víctima de un delito). En cambio, los participantes de la muestra de Venezuela, El Salvador y Bolivia son los que muestran puntajes más altos en diferentes ítems del miedo difuso. En esta línea, Cruz (2010) señala que el nivel de percepción de policía involucrada en delitos es la principal variable - junto con ser mujer- que juega en contra del apoyo a la democracia en la región latinoamericana.

En cuanto al primer objetivo, acerca de las relaciones entre el miedo al crimen y la satisfacción con la Policía, se encuentran relaciones significativas e inversas entre ambas variables, en la línea encontrada por otros estudios (por ejemplo, Bourne, 2016). Sin embargo, esta relación se haya en solo seis de las doce muestras. Ello muestra que la relación entre ambas variables es compleja, como lo destaca este último autor: una variable puede ser predictora de la otra, influida por aquella, de mutua influencia o de nula relación. Además, en caso de relaciones entre ambas, aunque en general se postula una mayor satisfacción con la Policía asociada a un menor miedo al delito, también puede darse que una mayor presencia de la Policía en un sector genere más temor, porque es interpretada como señal de que hay actividad criminal importante en ese área (Meško et al., 2007); incluso algún autor señaló la posibilidad de que la misma Policía tenga interés en generar un sentimiento de inseguridad alto para que la ciudadanía reclame y apoye destinar más recursos para combatir el crimen (Moore \& Trojanowicz, 1988). El conjunto de resultados sobre las relaciones entre miedo al crimen, satisfacción con la Policía y la victimización encontrado en este trabajo podría poner en cuestión el modelo de las actitudes punitivas y de búsqueda de justicia por mano propia basado en un nivel alto de miedo, la victimización y la baja satisfacción con la Policía.

En relación con el segundo objetivo, los resultados son más llamativos aún: se encuentran correlaciones significativas entre victimización y percepción sobre la Policía en solo cuatro de las muestras, y dos de estas son correlaciones directas, es decir, a mayor satisfacción con la Policía mayor victimización, y dos inversas: a mayor satisfacción con la Policía menor victimización, en la línea de lo encontrado por autores como Moore y Trojanowicz (1988) o Bahena (2015). Ello podría explicarse por las dinámicas particulares de las sociedades de donde proceden las muestras: las correlaciones directas en las muestras de España y de México podrían indicar que las víctimas encuentran una adecuada atención a sus demandas, de ahí la satisfacción con la Policía, mientras que la victimización en Colombia y Venezuela llevaría a una inconformidad con la actuación de la Policía en los aspectos medidos por el instrumento sobre la Policía en este trabajo. Empero, ello no explica la falta de relación significativa entre ambas variables en la mayoría de las muestras.

En cuanto al tercer objetivo, se halla una relación significativa y directa entre mayor victimización y mayor miedo al delito, pero, de nuevo, en solo cinco de las doce muestras. Al respecto,Alda et al. (2017) también hallaron un resultado 
similar en muestras aleatorias de siete naciones caribeñas. Ahora, los datos fueron analizados para el total de la muestra, sin separar por países, por lo que no es posible afirmar que los resultados se dieran de forma similar en cada nación, a diferencia de este trabajo en el cual se ofrecen resultados por cada una de las doce muestras nacionales que conforman la muestra total. Así, una mayor victimización se asociaría con un mayor miedo al crimen, pero no siempre ni en todo lugar, como advierte Bourne (2016).

Por otro lado, Alda et al. (2017) encontraron una relación directa entre indicadores comunitarios como la cohesión social, la tasa de pobreza, la tasa de crimen en el barrio (diferente de la victimización experimentada personalmente) con el miedo al crimen y la percepción sobre la Policía. En este estudio, se incluyó una medida de índole comunitario (la percepción de la eficacia colectiva). En otros trabajos, se han hallado relaciones entre mayor eficacia colectiva y menor miedo al crimen y victimización, y con mayor satisfacción con la Policía, en sujetos colombianos (Ruiz, 2015). En el actual estudio, no se encontraron relaciones entre miedo, victimización y eficacia colectiva, pero sí una correlación directa entre esta y la satisfacción con la Policía, en tres de las cinco aplicaciones que incluyeron la escala de eficacia colectiva.

Por último, a la luz de las relaciones y no relaciones encontradas entre las variables de interés en este trabajo, a modo tentativo se analizó el papel mediador de la victimización entre el miedo al crimen como variable predictora y la satisfacción con la Policía, tras lo cual se encontró un resultado significativo que apuntaría a la victimización como un factor que potencia moderadamente la relación inversa entre el miedo al crimen y la satisfacción con la Policía.

Este resultado podría ser abordado con mayor profundidad en futuras investigaciones que podrían incluir asimismo medidas más amplias sobre los componentes de lo que se ha denominado aquí percepción o satisfacción con la Policía. Además, se precisa el desarrollo de un modelo psicosocial sobre la Policía que articule y delimite conceptos relacionados pero diferentes como a) actividades de la Policía, b) confianza en la Policía, c) percepciones sobre la eficacia, velocidad de reacción, honestidad, etc., d) satisfacción con la Policía;y esto en su posible relación diferencial con medidas de miedo al crimen y la victimización objetiva, todo ello con muestras más amplias y equivalentes por contextos, para controlar posibles sesgos de las muestras. Este modelo, y los problemas conceptuales que involucra, se complejizan si se tiene en cuenta el problema de la medición del miedo al crimen. En este sentido, se han propuesto instrumentos de medición del miedo al crimen más recientes que los empleados en este trabajo (Reyes-Sosa \& Molina-Coloma, 2018), aunque con escasos datos de validación en muestras latinoamericanas y que no resuelven el debate acerca de si miden solo miedo al crimen o variables conectadas pero diferentes del miedo propiamente dicho.

\section{Referencias}

Alda, E., Bennett, R. R. \& Morabito, M. S. (2017). Confidence in the police and the fear of crime in the developing world. Policing: An International Journal of Police Strategies \& Management, 40(2), 366-379. https://doi.org//0.1 108/PIJPSM-03-2016-0045

Aparicio, J. (2017). El proceso de amalgamiento, centralización y unificación de los cuerpos de policía en los países de América. Bogotá: Universidad Externado de Colombia.

Bahena Rivera,A. (2015). Victimización, percepción de inseguridad y satisfacción con la vida en contextos de alta criminalidad: un análisis psicosocial en el estado de Morelos (Tesis doctoral, Universidad Pablo Olavide, Sevilla, España). Recuperado de https:// www.uv.es/lisis/otras-publica/tesis_alejandro_bahena.pdf

Bourne, B. (2016). Fear of crime and it's effect upon opinions of the police related to police professionalism and police efficiency: $A$ secondary data analysis. Albany, EE. UU.: State University of New York.

Carroll, J. M., Rosson, M. B. \& Zhou, J. (2005). Collective efficacy as a measure of community. Trabajo presentado en $\mathrm{CHI}$ '05 Proceedings of the SIGCHI Conference on Human Factors in Computing Systems, Portland, Oregon, EE. UU.

Casas, K., González, P.y Mesías, L. (2018). La transformación policial para el 2030 en América Latina. Washington DC, EE. UU.: Banco Interamericano de Desarrollo.

Cruz,J.M. (2010). Mala conducta policial y democracia en América Latina. Perspectivas desde el Barómetro de las Américas, 33, I-6. Recuperado de https://www.vanderbilt.edu/lapop/ insights/10833es.pdf

Domanick,J. (2016). Asegurando la confianza: una guía de mejores prácticas para comunicadores de cuerpos policiales que América Latina y el Caribe. Nueva York, EE. UU.: Banco Interamericano de Desarrollo.

elPeriódico. (2019, agosto 9). Guatemala, entre los países de mayor tránsito de drogas. Recuperado de https:// elperiodico.com.gt/nacion/2019/08/09/guatemala-entrepaises-de-mayor-transito-de-drogas/ 
Espinosa-Poveda, A. C. \& Ruiz, J. I. (20I2). Eficacia colectiva en Colombia. Social y Jurídica, I, 47-53.

Focás, B. (2018). Miedo al crimen, prevención del delito y narcotráfico: desafíos para las políticas públicas de seguridad ciudadana en América Latina. Entrevista a Lucía Dammert. Urvio: Revista Latinoamericana de Estudios de Seguridad, 22, I02-I08. http://dx.doi.org/|0.17|4|/urvio.22.2018.3 |83

Johnson, R. R. (2016). Reducing fear of crime and increasing citizen support for police. Recuperado de https://www.dolanconsultinggroup.com/wp-content/uploads/2016/07/ Research_Brief_Reducing-Fear-of-Crime-and-IncreasingCitizen-Support_July262.pdf

Latinobarómetro (2018). Informe 20/8. Recuperado http:// www.latinobarometro.org/latNewsShow.jsp

Ledesma, R., Macbeth, G. \& de Kohan, N. C. (2008). Tamaño del efecto: revisión teórica y aplicaciones con el sistema estadístico ViSta. Revista Latinoamericana de Psicología, 40(3), 425-439. http://publicaciones.konradlorenz.edu.co/index. php/rlpsi/article/download/358/257

Martínez-Zelaya, G., Reyes, H., Muratori, M. y Ruiz, J. I. (20I4). Estrategias de afrontamiento, valores personales, bienestar social y clima emocional: un estudio transcultural. Trabajo presentado en XII Congreso Nacional de Psicología Social, I Congreso de la SCEPS, Sevilla, España.

Meško, G., Fallshore, M., Rep, M. \& Huisman, A. (2007). Police efforts in the reduction of fear of crime in local communities: Big expectations and questionable effects. Sociologija: Mintis ir veiksmas, 2(20), 70-9I. Recuperado de https:// www.ceeol.com/search/article-detail?id $=200130$

Moore, M. H. \& Trojanowicz, R. C. (1988). Policing and the fear of crime. Perspectives in Policing, 3, 1-8. Recuperado de https://pdfs.semanticscholar.org/dc45/I63ebd Ib I986697e e9f9/913b727f09ba673.pdf

Páez, A. D. y Ruiz, J. I. (20I2). Miedo al crimen en Colombia. Social y Jurídica, I, 60-66.

Páez Murillo, C. A., Peón Escalante, I. E. y Ramírez Pedraza, Y. (2018). Contexto de la seguridad ciudadana en América Latina y el Caribe. Revista Científica General José María Córdova, I6(24), 83-106. https://doi.org/I0.2 I830//9006586.360
Reyes-Sosa, H.y Molina-Coloma, H. (20I8).Análisis psicométrico de una escala para medir el miedo al delito en jóvenes ecuatorianos. Acta Colombiana de Psicología, 2 I ( I), 300-309. DOI: 10.147I8/ACP.2018.21.I.I3.

Ruiz, J. I. (2007a). Policía y construcción de cultura ciudadana: un estudio exploratorio. Cuadernos Hispanoamericanos de Psicología, 6(2), 143-150.

Ruiz, J. I. (2007b). Procesos sociales relacionados con el miedo al crimen, la satisfacción con la policía y la victimización: el caso de la cultura ciudadana. International E-Journal of Criminal Sciences, I, I-29. Recuperado de https://dialnet.unirioja. es/servlet/articulo?codigo $=4876031$

Ruiz, J. I. (20I4). Psicología social y justicia. Bogotá, Colombia:Universidad Nacional de Colombia.

Ruiz, J. I. (20I5). Diplomado en capacidades ciudadanas: explorando la eficacia colectiva. Bogotá, Colombia: Universidad $\mathrm{Na}$ cional de Colombia.

Ruiz, J. I. y Turcios, L. A. (2009). Percepción de seguridad, victimización y cultura ciudadana: sus relaciones en cinco contextos iberoamericanos. Pensamiento Psicológico, 6(13), 193-202. Recuperado de https://www.redalyc.org/ pdf/80 I/80 I I 24690 I 3.pdf

Ruiz, J. I., López, E. J., Norza, E. \& Rodríguez, L. S. (2012). Monográfico: Percepción de seguridad en jóvenes colombianos. Social y Jurídica, 2.

United Nations Office on Drugs and Crime. (2019). Global Study on Homicide 2019.Viena, Austria:Autor. Recuperado de https://www.unodc.org/documents/data-and-analysis/gsh/ Booklet2.pdf

Weisburd, D. \& Eck, J. E. (2004).What can police do to reduce crime, disorder, and fear? The Annals of the American Academy of Political and Social Science, 593( I), 42-65. https://doi. org/I0.I I 77/00027/6203262548 